\title{
Doppler-based Motion Compensation Strategies for 3D Diverging Wave Compounding and Multi-Plane-Transmit Beamforming: A Simulation Study
}

\author{
Yinran Chen, Student Member, IEEE, Jan D'hooge, Member, IEEE, and Jianwen Luo, Senior Member, \\ IEEE
}

\begin{abstract}
Fast imaging of the heart has shown promise towards bringing new diagnostic information. Although most studies to date have been based on two-dimensional (2D) imaging technology, the ultimate diagnostic tool would enable fast 3D echocardiography. Hereto, 3D diverging wave compounding (DWC) and 3D multi-line-transmit (MLT) beamforming have recently been proposed. Moreover, in our recent study, a hybrid technique was proposed in which multiple planar diverging waves were transmitted (i.e., multi-plane-transmit, MPT). The proposed 3 MPT sequence was demonstrated to outperform $9 \times 9 \mathrm{DWC}$ and 16MLT-4MLA (i.e., multi-line-acquisition) while imaging moving targets. However, none of the investigated beamforming techniques made use of motion compensation (MoCo) strategies. In this paper, we therefore propose Doppler-based MoCo strategies for 3D DWC and MPT and test them via computer simulations. It is demonstrated that the MoCo strategies proposed for both DWC and MPT are effective and significantly restore image quality. Moreover, the MPT beamforming with MoCo outperforms $9 \times 9 \mathrm{DWC}$ with MoCo in terms of contrast ratio (CR) and contrast-to-noise ratio (CNR). The proposed MPT beamforming with MoCo thus provides volumetric images with relatively high temporal resolution $(\sim 66 \mathrm{~Hz})$ and high image quality that is minimally affected by motion artifacts.
\end{abstract}

Index Terms-3-D ultrasound imaging, diverging wave beamforming, motion compensation, multi plane transmit (MPT), volumetric imaging.

\section{INTRODUCTION}

Ultrasound imaging is a common choice for clinical diagnosis for the heart given its advantages over other imaging modalities, such as high accessibility, low cost, free of ionizing radiation and real-time operation. Conventional 2D echocardiography is based on single-line-transmit (SLT) and

Manuscript received xxxx xx, 2017. The research leading to these results has received funding from the National Key R\&D Program of China (2016YFC0104700 and 2016YFC0102200), the National Natural Science Foundation of China (NSFC) (81471665 and 81561168023).

Y. Chen is with the Department of Biomedical Engineering, School of Medicine, Tsinghua University, Beijing 100084, China.

J. D'hooge is with the Laboratory on Cardiovascular Imaging and Dynamics, Department of Cardiovascular Sciences, KU Leuven, 3000 Leuven, Belgium.

J. Luo is with the Department of Biomedical Engineering, School of Medicine, Tsinghua University, Beijing 100084, China (e-mail: luo_jianwen@tsinghua.edu.cn). beam scanning, leading to a temporal resolution of $\sim 25$ to 40 $\mathrm{Hz}$, which is insufficient to capture the transient phases of cardiac motion, e.g., isovolumetric contraction, isovolumetric relaxation, and fast propagation associated with electromechanical depolarization or shear waves (typically in a range of 1-10 m/s) [1]-[5]. Moreover, out-of-plane structures and motion are inaccessible due to $3 \mathrm{D}$ nature of the heart. Furthermore, image quality can be deteriorated by motion artifacts caused by fast myocardial motion in combination with relatively low temporal resolution. To overcome these limitations, developing advanced 3D echocardiographic techniques with relatively high temporal resolution and high image quality, as well as free of motion artifacts, recently become a research hot-topic [6]-[8].

The main idea of increasing temporal resolution in echocardiography attempts to reduce the amount of transmit events, as the intrinsic acoustic velocity in tissues, the imaging depth, and the demanded field-of-view (FOV) are less adjustable. There are two major solutions to high-frame-rate echocardiography, i.e., multi-line-transmit (MLT) and diverging wave compounding (DWC) [9] , as will be discussed in the following paragraphs.

MLT beamforming is based on transmitting multiple focused beams simultaneously, with each beam covering a respective sub-sector [10]-[12]. The frame rate of MLT can be increased by a factor equal to the number of MLT beams when compared with the conventional line-by-line scanning. Moreover, when combining MLT with parallel beamforming in receive (i.e., multi-line-acquisition, MLA), the frame rate is further increased by another factor equal to the number of MLA beams. Meanwhile, the potential crosstalk artifacts resulting from the interaction between the MLT beams can be significantly suppressed by different means [11], [13]-[20]. Recently the MLT-MLA setup has been extended to $3 \mathrm{D}$, where the image quality is mostly preserved and the acquisition process can be sped up by a factor of, for example, 36 (i.e., 9MLT-4MLA) and thus a full field-of-view at a volume rate of $\sim 32 \mathrm{~Hz}$ with conventional line density can be achieved [21], [22].

In analogy with SLT, MLT beamforming suffers from image distortions caused by the relative motion between transducer and tissues due to the scan process [23]. Furthermore, stitching 
artifacts can be observed at the edges of adjacent sub-sectors generated by MLT beams in an one-side-to-another sequential scan scheme [12]. The image distortions can be restored by conventional tissue Doppler methods [24]. The stitching artifacts can be significantly reduced by adapting the sequence to scan from the sub-sector edges to the center in order to evenly distribute the time lags between recordings of neighboring lines [12], [14].

As an alternative to MLT, the imaging region can be covered in one shot by transmitting plane/diverging waves, thus leading to a very high frame rate (i.e., ultrafast ultrasound imaging) [25, 26]. However, in order to avoid too much loss in image quality, spatial coherent compounding is typically needed thereby compromising the effective gain in frame rate (e.g., diverging wave compounding, DWC) [27]-[30]. This technique has also been demonstrated to be extendable to $3 \mathrm{D}$ to provide high volume rate image with relatively high image quality [31], [32].

DWC imaging suffers from different motion artifacts, showing loss in spatial and contrast resolution caused by phase incoherence in the compounding operation [33], [34]. Several MoCo strategies have previously been investigated by using auto-correlation or cross-correlation in plane or diverging wave compounding imaging [23], [34]-[46]. In particular, a modified Doppler-based MoCo strategy is applied to 2D echocardiography with steered diverging waves being transmitted in a "triangle" sequence in order to eliminate the side effects induced by rotation of the point spread functions (PSFs) [44]. Very recently, this technique is also demonstrated to be feasible in $3 \mathrm{D}$, with several steered spherical diverging waves transmitted in a dedicated scan sequence [46].

Finally, a hybrid beamforming technique combining the features of MLT and DWC is proposed in our previous work, where multiple planar diverging waves are transmitted in 3D simultaneously (i.e., multi-plane-transmit, MPT) [47]. In particular, the MPT beams behave as 2D DWC in the azimuth dimension but as MLT in the orthogonal dimension. Although it is demonstrated to outperform 3D MLT and 3D DWC in imaging of moving targets, MoCo is not taken into account.

The objective of this study is therefore to propose Doppler-based MoCo strategies in 3D DWC and MPT respectively, in order to provide volumetric B-mode images of good image quality and high volume rate that are minimally affected by motion artifacts. For 3D DWC, a series of diverging waves are transmitted in a "round-trip" scan sequence and several sequences are investigated to find the optimal one in cooperation with the MoCo strategy. For MPT, the MoCo strategy proposed in [44] is applied to correct motions within the transmit beam planes with subsequent MoCo in between the beam planes. Finally, both beamforming sequences with MoCo are directly contrasted in terms of their resulting image quality. All this is studied by computer simulations. Abbreviations used in this paper are summarized in Table I.
TABLE I

ABBREVIATIONS

\begin{tabular}{cc}
\hline \hline Short name & Full name \\
\hline $2 / 3 \mathrm{D}$ & Two/ three-dimension(al) \\
SLT & Single-line-transmit \\
MLT & Multi-line-transmit \\
MLA & Multi-line-acquisition \\
DWC & Diverging wave compounding \\
CMP & Compounding \\
MPT & Multi-plane-transmit \\
MoCo & Motion compensation \\
CR & Contrast ratio \\
CNR & Contrast-to-noise ratio \\
SNR & Signal-to-noise ratio \\
\hline \hline
\end{tabular}

TABLE II

TRANSDUCER CONFiguration Of THE Matrix ARRAy

\begin{tabular}{cc}
\hline \hline Parameters & Values \\
\hline No. elements (azi $\times$ ele) & $32 \times 32$ \\
Element size & $0.30 \mathrm{~mm} \times 0.30 \mathrm{~mm}$ \\
Pulse center frequency & $3.0 \mathrm{MHz}$ \\
Pulse length & $1.5 \mathrm{cycles}$ \\
Bandwidth & 0.8 \\
\hline \hline
\end{tabular}

azi $=$ azimuth, ele $=$ elevation.

TABLE III

BEAMFORMING SETUP

\begin{tabular}{cc}
\hline \hline Parameters & Values \\
\hline Speed of sound & $1,540 \mathrm{~m} / \mathrm{s}$ \\
Pulse repetition frequency & $5 \mathrm{kHz}$ \\
Sampling frequency & $100 \mathrm{MHz}$ \\
for Field II simulation & \\
Sampling frequency & $25 \mathrm{MHz}$ \\
for Rx beamforming & $2 \mathrm{D}$ Tukey $(\alpha=0.5)$ \\
Tx-Rx apodizations & Delay-and-sum \\
Rx beamforming & Dynamic focusing \\
& Rectangular pyramid \\
Imaging volume & $90 \times 90$ lines \\
& $60^{\circ} \times 60^{\circ}$ opening angle \\
& Azimuth-axial \\
Display cross-sections & Elevation-axial \\
& Diagonal dimension \\
Display dynamic range & C-plane view \\
\hline \hline
\end{tabular}

\section{METHODS}

\section{A. Simulation Setup}

1) Transducer Configuration and Beamforming Setup All simulations were carried out in Field II [48], [49]. A matrix array of 32-by-32 elements and a center frequency of 3 $\mathrm{MHz}$ was used. The configuration parameters of this transducer are given in Table II. In the beamforming process, the pulse repetition frequency (PRF) was set to $5 \mathrm{kHz}$. 2D Tukey $(\alpha=0.5)$ window functions were applied in transmit/receive $(\mathrm{Tx} / \mathrm{Rx})$ apodizations in order to suppress potential crosstalk artifacts [11]. In receive, the delay-and-sum beamforming with dynamic focusing and full-aperture-receive was used to reconstruct a pyramidal volume. The volume contained 90 -by-90 image lines and opening angles of $60^{\circ}$-by- $60^{\circ}$ in the azimuth and elevation dimensions. To evaluate the image properties of the 


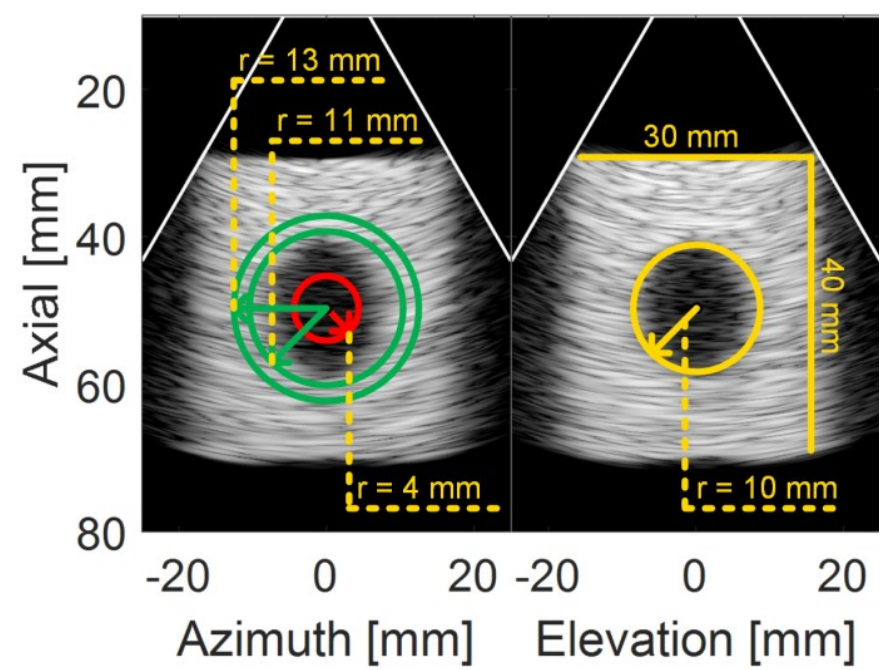

Fig. 1. Illustrations of imaging object and quantitative analysis methods. The imaging object is a cubic phantom (size: $30 \mathrm{~mm} \times 30 \mathrm{~mm} \times 40 \mathrm{~mm}$ ) containing a spherical cystic region (radius: $10 \mathrm{~mm}$ ). For quantitative analysis of contrast ratio $(\mathrm{CR})$ and contrast-to-noise ratio (CNR), the sphere in red is defined as the cystic region (radius: $4 \mathrm{~mm}$ ), while the spherical shell in green is defined as the background region (inner radius: $11 \mathrm{~mm}$, outer radius: $13 \mathrm{~mm}$ ).

reconstructed volumes, five representative cross-sections, i.e., the azimuth-axial plane, the elevation-axial plane, the two planes along the diagonal dimension, and a C-plane at a depth of $50 \mathrm{~mm}$, were displayed with a $55 \mathrm{~dB}$ dynamic range. All the beamforming parameters are summarized in Table III.

2) Imaging Objects and Quantitative Analysis

The imaging object was a $30 \mathrm{~mm} \times 30 \mathrm{~mm} \times 40 \mathrm{~mm}$ (azimuth $\times$ elevation $\times$ axial) cubic phantom containing a spherical cystic region with a radius of $10 \mathrm{~mm}$, as illustrated in Fig. 1. The scatterers forming this phantom had a density of 5 $/ \mathrm{mm}^{3}$, which was sufficient to generate fully developed speckle. Their scattering amplitudes were uniformly distributed between 0 and 1 outside the cystic region and set to 0 inside the cystic region.

For quantitative analysis, the contrast ratio (CR) and contrast-to-noise ratio (CNR) of the cystic region were calculated as follows:

$$
\begin{gathered}
C R=\mu_{\text {back }}-\mu_{c y s t} \\
C N R=20 \times \log _{10}\left(\frac{\mu_{\text {back }}-\mu_{c y s t}}{\sqrt{\sigma_{\text {back }}^{2}+\sigma_{c y s t}^{2}}}\right)
\end{gathered}
$$

Where $\mu_{\text {back }}$ and $\mu_{c y s t}$ were the mean image data (after log-compression) in the background and cystic regions as indicated in Fig. 1, respectively. And $\sigma_{\text {back }}$ and $\sigma_{c y s t}$ were their respective standard deviations.

To investigate the performance of the proposed MoCo strategies (mentioned in the following sections), the phantom was moved in the axial direction at a speed of $10 \mathrm{~cm} / \mathrm{s}$. This velocity was chosen as a realistic value for myocardial motion [50]. In addition to these simulations, the phantom was also imaged in a static state as benchmark. Furthermore, in the performance comparison of MPT and DWC, noise at different signal-to-noise ratio (SNR) levels was added before MoCo in order to test the robustness of the proposed techniques to noise.

\section{B. MPT Implementations with MoCo}

1) Principle of MPT Beamforming

The time delays to generate one of the MPT beams is illustrated in Fig. 2(a3). In order to generate a planar diverging wave using a matrix array, we need to make the beam diverging in one dimension (e.g., azimuth), as illustrated in Fig. 2(a1), and focused in the orthogonal dimension (e.g., elevation), as illustrated in Fig. 2(a2). As a result, the time delays in Fig. 2(a3) are simply the sum of the delays in Fig. 2(a1) and Fig. 2(a2) [47]. The simulated pressure field corresponding to time delays in Fig. 2(a3) in a C-plane view at a depth of $50 \mathrm{~mm}$ is shown in Fig. 2(b1). It is thus feasible to generate a beam that is diverging in the azimuth dimension, and focused in the elevation dimension. Moreover, in analogy with MLT, simultaneously transmitting multiple planar diverging waves into different directions requires the superposition of the time delays of several MPT beams focused at different directions in the elevation dimension. The simulated pressure field of a 3MPT case is shown in Fig. 2(b2).

The PSFs of different 3D beamforming techniques are summarized in Fig. 2(c). The PSF of 1MPT-5CMP (i.e., single planar diverging wave transmit with 5 compounds in the azimuth dimension, CMP = compounding), as shown in Fig. $2(\mathrm{c} 3)$, resembles that of $5 \times 5 \mathrm{DWC}$ in the azimuth dimension, while it looks like the PSF of the 3D focused wave beamforming in the elevation dimension, as illustrated in Fig. 2(c1) and 2(c2) respectively. In the 3MPT-5CMP case (i.e., 3 planar diverging waves transmitted simultaneously, with 5 compounds in the azimuth dimension), crosstalk artifacts occur as illustrated in Fig. 2(c4).

2) Scan Sequence of MPT

A 3MPT-2MLA-5CMP sequence was setup in this study, as illustrated in Fig. 3. 3MPT represented 3 planar diverging waves in a fixed inter-beam space of $20^{\circ}$ being transmitted simultaneously [see Fig. 2(b2) and Fig. 3]; 2MLA represented parallel receive beamforming around each MPT implying that two 2D images were reconstructed for each MPT beam (i.e., there were six 2D images reconstructed in parallel in one 3MPT transmission event) [see Fig. 3(b)]; 5CMP represented the 3MPT beams being transmitted repetitively while moving the transmit aperture in the azimuth dimension in order to enable in-plane compounding of these 5 planar diverging waves [see Fig. 3(a)].

In order to perform MoCo in MPT, a "round-trip" scan sequence was implemented in the azimuth dimension, as illustrated in Fig. 3(a) and 3(c1). Hereto, the 3MPT beams were simultaneously transmitted repetitively while sliding the transmit sub-aperture in the azimuth dimension according to a "round-trip" compounding scheme, without changing the beam focusing directions or the inter-beam spacing in the elevation dimension. Then this process was repeated while sweeping the MPT beams in the elevation dimension in an "edge-to-center" sequence, as illustrated in Fig. 3(c2), i.e., the MPT beams were scanned from the sub-sector edges to the centers as this more evenly distributes the time lag between recordings of 


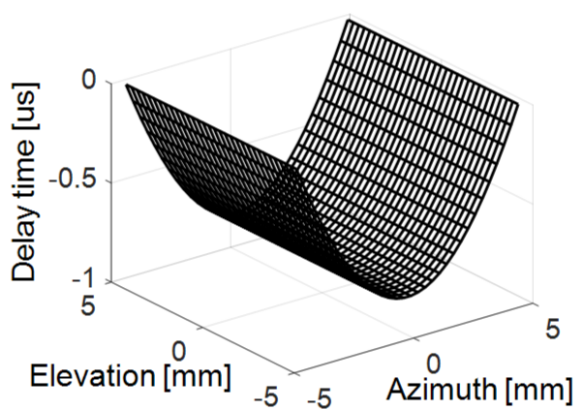

(al)

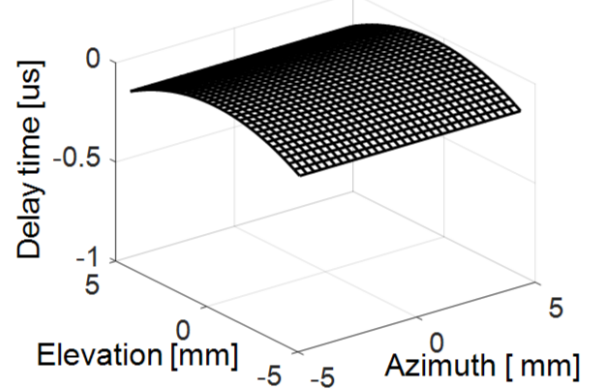

(a2)

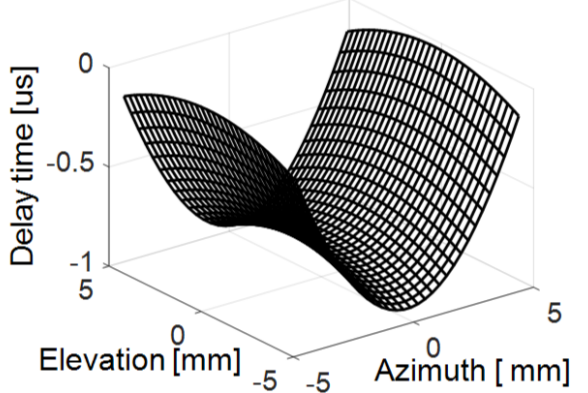

(a3)

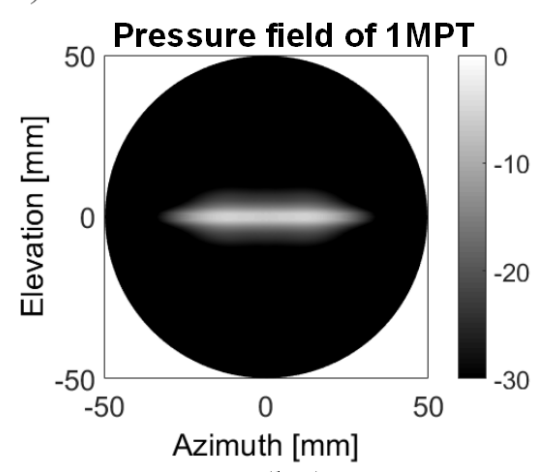

(bl)

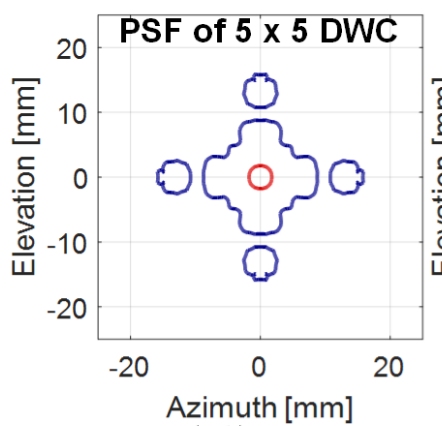

(cl)

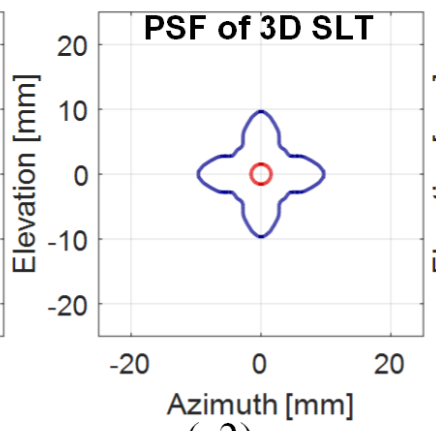

(c2)

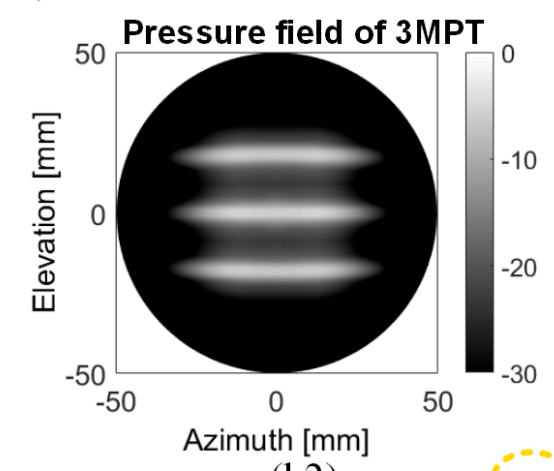

(b2)

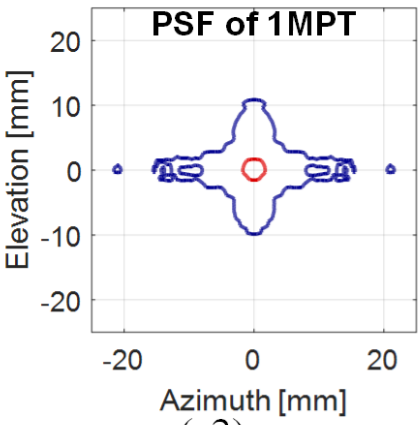

(c3)

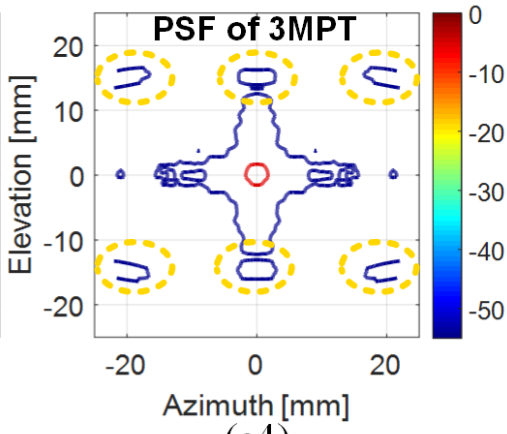

(c4)

Fig. 2. The principle of MPT beamforming. (a) Generation of transmit time delays for one of the MPT beams, with (a1) showing the time delay component to generate diverging wave in the azimuth dimension, (a2) The time delay component to generate focused wave in the elevation dimension, and (a3) the time delays for generation of one planar diverging wave, which is the summation of (a1) and (a2). (b1) The simulated transmit pressure field of one of the MPT beams, corresponding to the time delays in (a3). (b2) The simulated transmit pressure field of the $3 \mathrm{MPT}$ case. The generation of the time delays of 3MPT requires the superposition of the time delays focused at different directions. (c) The PSFs of different 3D beamforming from a C-plane view at depth of 50 mm, with (c1) PSF of the $5 \times 5$ DWC sequence, (c2) PSF of the 3D SLT sequence, (c3) the PSF of the 1MPT-5CMP sequence, and (c4) the PSF of the 3MPT-5CMP sequence. The PSF profile in (c3) resembles that of (c1) in the azimuth dimension, while it looks like that of (c2) in the elevation dimension. In (c4), crosstalk artifacts are highlighted by the yellow circles. The solid lines in red indicate the $-6 \mathrm{~dB}$ contour line, while the solid lines in blue indicate the $-55 \mathrm{~dB}$ contour line.

neighboring planes. This setup led to a total of 150 transmit events to cover the volume twice while meeting the requirement of the MoCo strategy: firstly, 10 transmissions in a "round-trip" sequence were made in the azimuth dimension without steering the MPT beams; secondly, the process above was repeated 15 times while sweeping the MPT beams in the elevation dimension (i.e., 90 image lines $\div 3 \mathrm{MPT} \div 2 \mathrm{MLA}=$ $15)$. As a results, the volume rate of the 3MPT-2MLA-5CMP setup was $\sim 66 \mathrm{~Hz}$ at a PRF of $5 \mathrm{kHz}$.

\section{3) MoCo Strategy}

The MoCo strategy for MPT consisted of 2 steps. In the $1^{\text {st }}$ step, MoCo was performed within the beam planes prior to compounding. This was a $2 \mathrm{D}$ operation where Doppler velocities were estimated in every pixel in each beam plane by using the following formulas [44]:

$$
\begin{gathered}
\theta_{\text {velocity }}=\frac{1}{2} \angle\left\{R_{\text {forward }} \times R_{\text {backward }}\right\} \\
V_{\text {Doppler }}=\frac{\theta_{\text {velocity }}}{2 \pi} \times \frac{P R F \times c}{2 \times f_{0}}
\end{gathered}
$$

where $R_{\text {forward }}$ and $R_{\text {backward }}$ were the lag-one auto-correlation coefficients in the forward and backward trip respectively, $\theta_{\text {velocity }}$ was the phase shift corresponding to the Doppler velocity, $P R F$ was the pulse repetition frequency, $c$ was the speed of sound $(1,540 \mathrm{~m} / \mathrm{s}), f_{0}$ was the pulse center frequency, and $V_{\text {Doppler }}$ was the estimated velocity. The length of axial gate in the Doppler estimation was around $0.4 \mathrm{~mm}$ (13 beamformed points at a sampling frequency of $25 \mathrm{MHz}$ ). No further post 


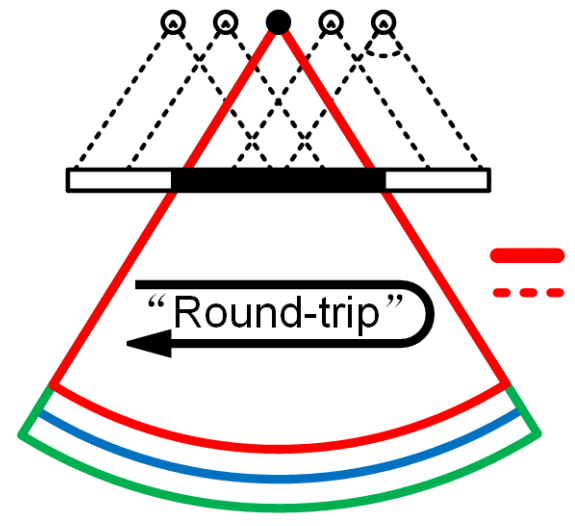

(a) Azimuth view
3MPT-2MLA-5CMP
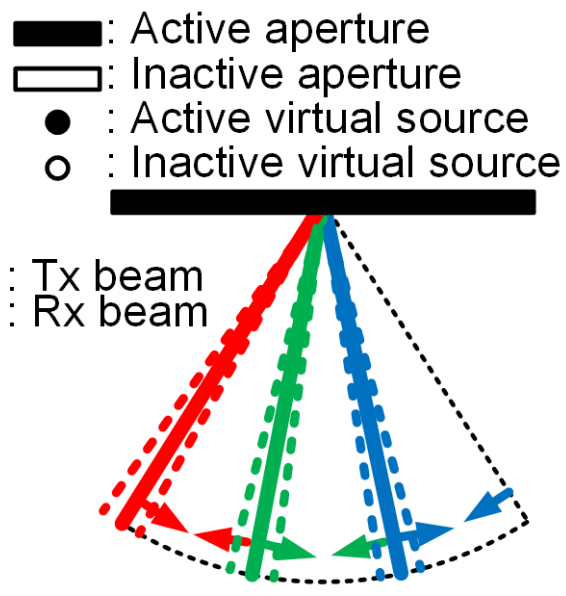

(b) Elevation view

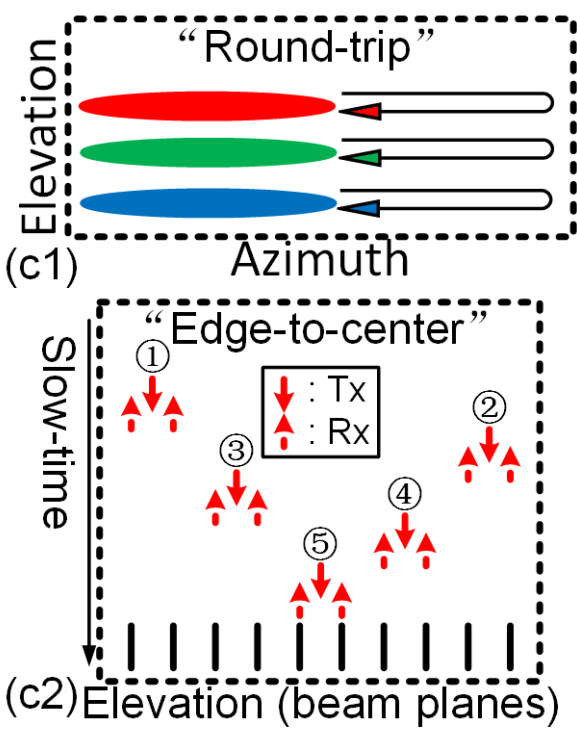

Fig. 3. Illustration of the 3MPT-2MLA-5CMP sequence. (a) View from the azimuth dimension, where the beams are diverging and behave as $2 \mathrm{D}$ diverging wave compounding. The size of the transmit sub-aperture in this dimension is equal to 16 elements. Compounding is performed by moving the sub-aperture without steering the beams. In this dimension, the MPT beams are in a "round-trip" scan sequence. (b) View from the elevation dimension, where the beams behave as MLT. The focused depth is $50 \mathrm{~mm}$ and the inter-beam space is $20^{\circ}$. The "Tx beam" and "Rx beam" indicate a parallel receive beamforming, i.e., 2MLA. Two 2D images surrounding one MPT beam are reconstructed simultaneously in receive. (c1) indicates the 3MPT beams from a top view, where the beams are transmitted simultaneously in a "round-trip" scan sequence in the azimuth dimension. (c2) indicates the "edge-to-center" scan sequence in the elevation dimension, i.e., the beams are transmitted from the edge to the center of the scanning sub-sector.

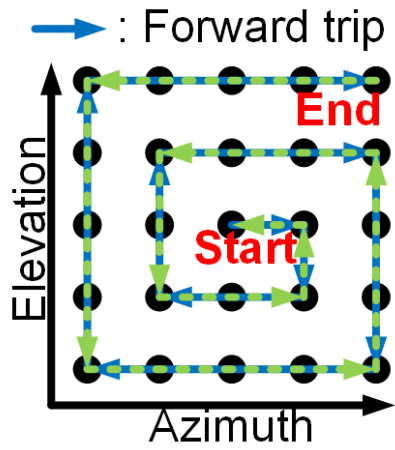

(a) Spiral

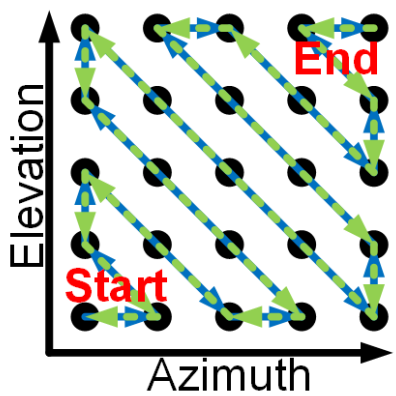

(c) Diagonal

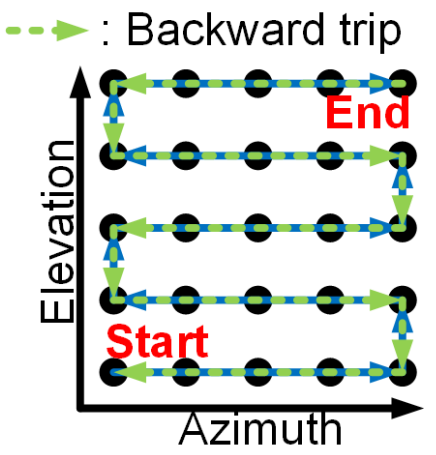

(b) Parallel

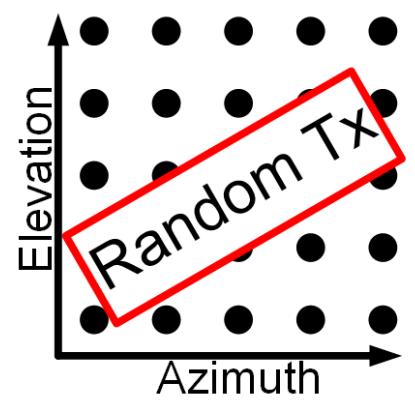

(d) Random
Fig. 4. Illustration of the "round-trip" scan sequence in a $5 \times 5$ DWC case. The virtual sources (i.e., the black spots) are sequentially activated in a specific sequence from the start point to the end point, and then they are transmitted back from the end point to the start point, following the original path. (a) The Spiral sequence. (b) The Parallel sequence. (c) The Diagonal sequence. (d) The Random sequence, where the virtual sources are sequentially activated randomly, but also in a "round-trip" scheme.

processing was applied on the Doppler estimates. The velocities were then used to correct the phase incoherence of the respective in-plane planar diverging waves.
In the $2^{\text {nd }}$ step, MoCo was applied to correct for the time lags between the neighboring planes in the elevation dimension. Hereto, the displacements of the neighboring planes were computed as the product of the Doppler velocity map and the time lag between the two planes. The cumulative displacements between the measured planes and the reference planes (i.e., the 6 planes acquired first) were estimated at a pixel level by adding the neighboring displacements together. Please note that such operation was performed in the 6 parallel acquisition planes.

\section{3D DWC Implementations with MoCo \\ 1) Scan Sequence of DWC}

A $9 \times 9$ DWC sequence with a transmit sub-aperture of 16-by- 16 elements and opening angle of $60^{\circ}$-by- $60^{\circ}$ was setup. The $9 \times 9$ compounding scheme was chosen in order to provide better spatial resolution as well as significant improvement in contrast resolution [31]. Such setup led to a volume rate of $\sim 61$ $\mathrm{Hz}$, which was comparable to that of the 3MPT-2MLA-5CMP setup (i.e., $\sim 66 \mathrm{~Hz}$ ), thus making the comparison fair.

2) MoCo Strategy

A "round-trip" scan sequence was designed in order to perform MoCo in 3D DWC, as illustrated in Fig. 4. More specifically, the virtual sources located behind the matrix array were sequentially activated from the start point to the end point in a dedicated sequence, then, very importantly, they were transmitted back from the end point to the start point, following the original path. The Doppler velocity of each beamformed voxel within this "round-trip" period was estimated based on (3) and (4), with the same axial gate length used for MoCo of MPT. Then, the displacements between the measured volumes and the reference volume (i.e., the volume corresponding to the first activated virtual source) were computed as the product of the 


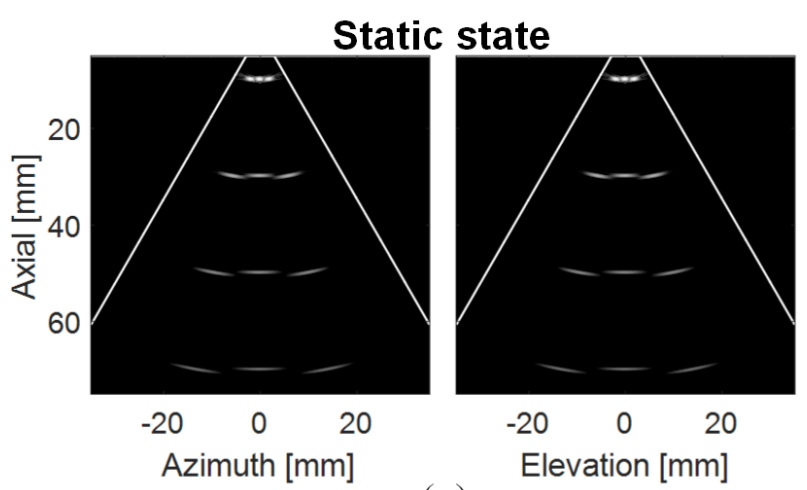

(a)

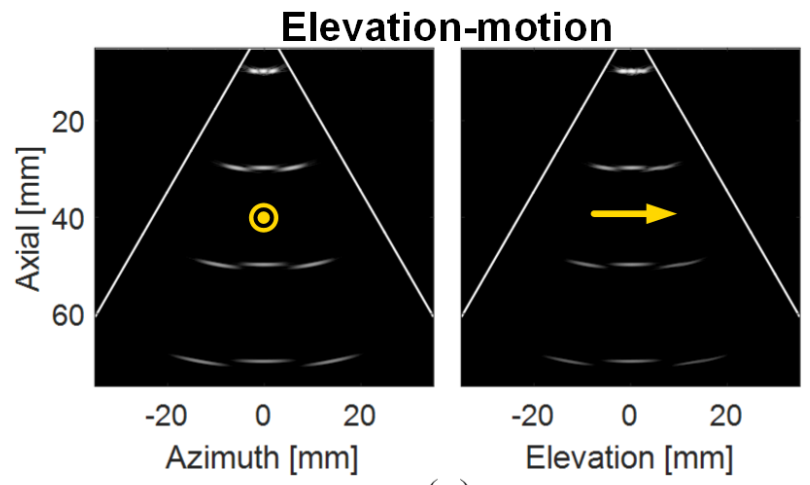

(c)

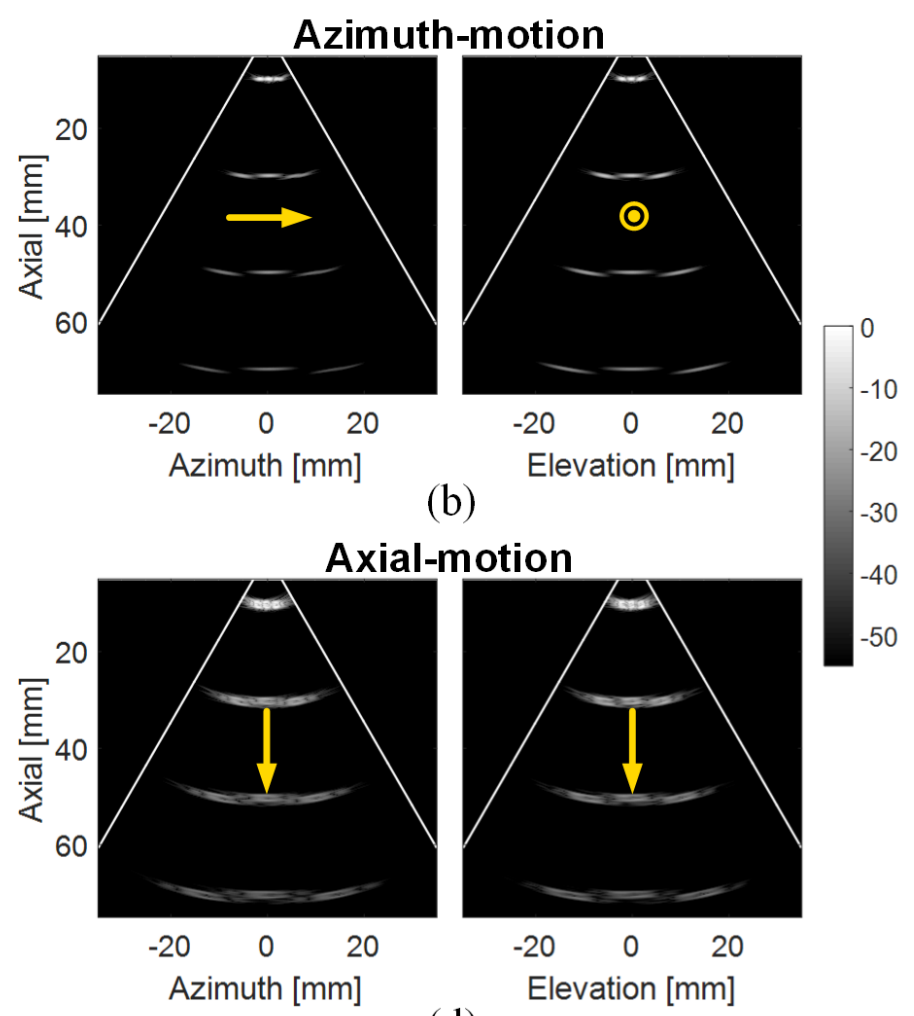

(d)

Fig. 5. Motion artifacts of PSFs with motion at a velocity of $10 \mathrm{~cm} / \mathrm{s}$ in different directions. (a) PSFs in the static state, shown as benchmark. (b) PSFs with motion in the azimuth direction. (c) PSFs with motion in the elevation dimension. (d) PSFs with motion in the axial direction. The yellow arrows and circles indicate the motion direction, with the circles representing the direction perpendicular to the image plane. Please note that no MoCo is applied in order to study the intrinsic motion artifacts.

Doppler velocities and the time lag. No further post processing was performed before or after MoCo.

3) Sequence Optimization

Four scan sequences (i.e., the Spiral, Parallel, Diagonal and Random sequences) were tested for optimization. The patterns of the respective sequences in a $5 \times 5$ DWC case are shown in Fig. 4. For the Random sequence, the virtual sources were sequentially activated randomly, but also in a "round-trip" scheme. In order to make the optimization more generalized and convincing, both $5 \times 5$ and $9 \times 9$ DWC were tested respectively with the four scan sequences.

\section{RESULTS}

\section{A. PSFs with Motion}

The results of the PSFs obtained using $5 \times 5$ DWC with the Diagonal sequence is illustrated in Fig. 5. The images in Fig. 5(a) show the PSFs in the static state, which are taken as benchmark. The images in Fig. 5(b), 5(c) and 5(d) show the PSFs with motion in the azimuth, elevation, and axial directions, respectively. The velocity was $10 \mathrm{~cm} / \mathrm{s}$ and the yellow arrows/circles indicate the direction of motion. It can be seen that the azimuth or elevation motion has little impact on the profiles of PSFs, as illustrated in Figs. 5(b) and 5(c), respectively. On the contrary, the axial motion causes severe phase incoherence on the PSFs, deteriorating image quality, as shown in Fig. 5(d).

\section{B. 3D DWC Sequence Optimization}

The images of the simulated cystic phantom using the $5 \times 5$ DWC with the Parallel sequence in the static state, the moving state without and with MoCo are presented in Fig. 6. The $1^{\text {st }}$ row shows the images of the phantom in the static state, which are taken as benchmark. The $2^{\text {nd }}$ row gives the images of the phantom in the moving state without MoCo, resulting in obvious motion artifacts. The $3^{\text {rd }}$ row presents the images of the moving phantom with MoCo, showing qualitatively that the image quality is restored.

Quantitatively, the CNRs and CRs of $5 \times 5$ and $9 \times 9$ DWC for the four tested scan sequences are summarized in Fig. 7(a) and 7(b), respectively. The red lines indicate the CNR/CR obtained with the stationary phantom as benchmark. The bars in dashed/solid line indicate the results without/with MoCo respectively. In the static state, $9 \times 9 \mathrm{DWC}$ shows slightly higher CNR and CR than $5 \times 5$ DWC. However, when motion is present and MoCo is applied, $5 \times 5$ DWC outperforms $9 \times 9$ DWC. Furthermore, the results of $5 \times 5$ and $9 \times 9$ DWC both show that the Diagonal sequence with MoCo obtains the best performance in terms of CNR and CR. As a result, the Diagonal sequence was chosen for comparison with MPT in the following sections.

\section{Comparison of MPT and DWC with MoCo}

The images of 3MPT-2MLA-5CMP and $9 \times 9$ DWC with the Diagonal sequence are presented in Fig. 8. The results of $9 \times 9$ 


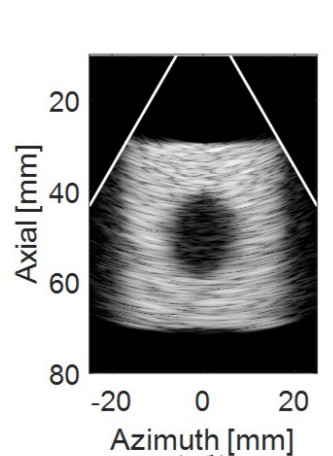

(al)

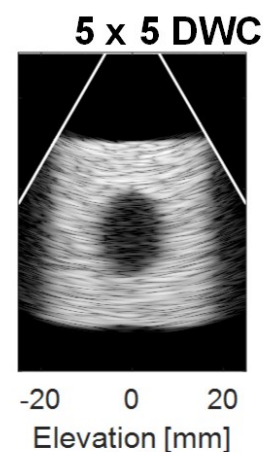

(a2)

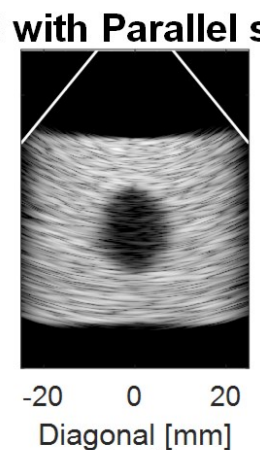

(a3)

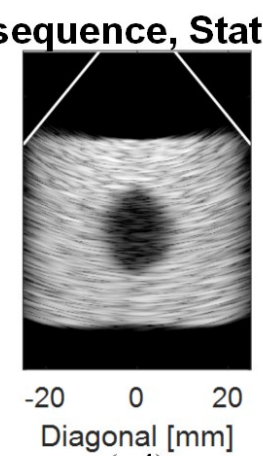

(a4)

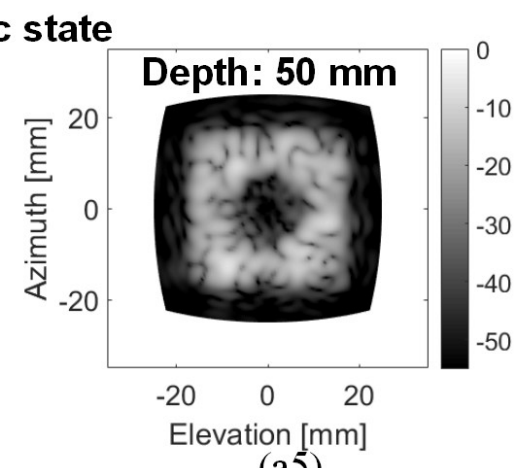

(a5)

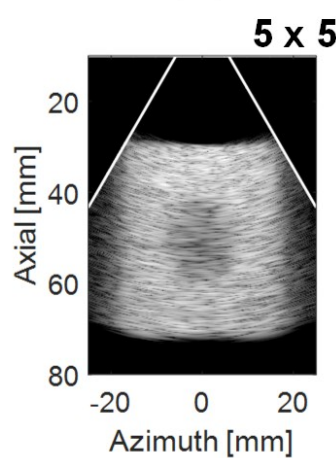

(b1)

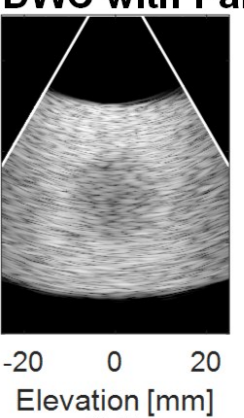

(b2)

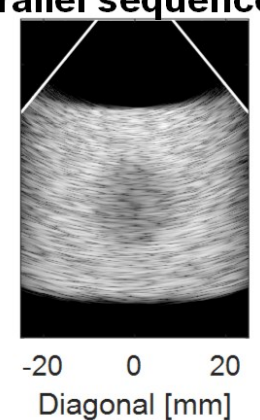

(b3)

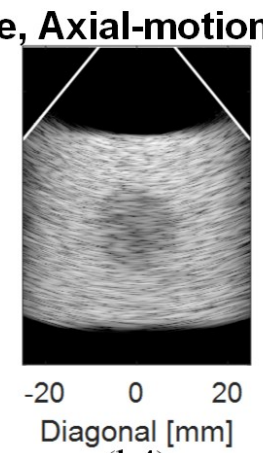

(b4)

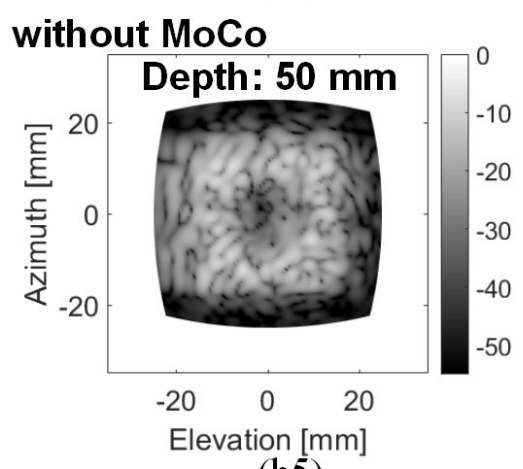

(b5)

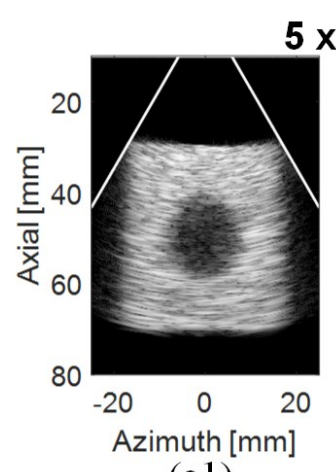

(c1)

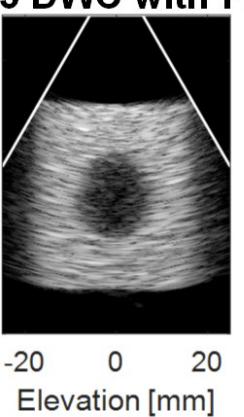

(c2)

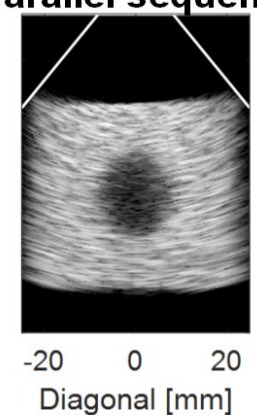

(c3)

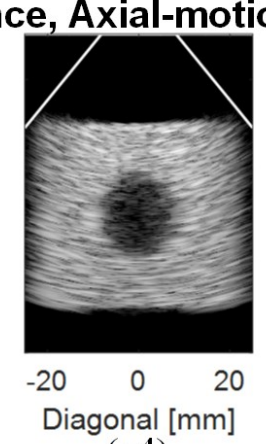

(c4)

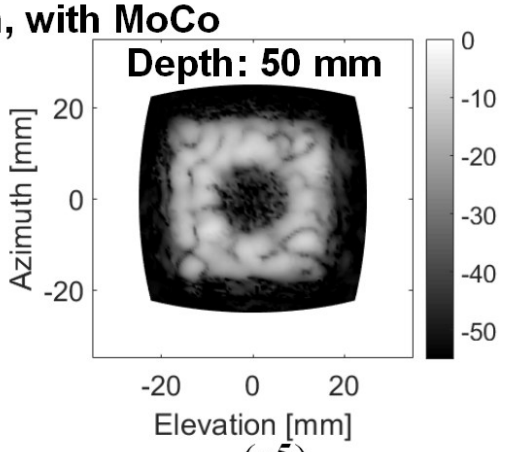

(c5)

Fig. 6. Images acquired by $5 \times 5$ DWC with the Parallel sequence in the static and moving state. The $1^{\text {st }}$ column shows the azimuth-axial plane, the $2^{\text {nd }}$ column the elevation-axial plane, the $3^{\text {rd }}$ and $4^{\text {th }}$ columns the two planes in the diagonal dimensions, and the $5^{\text {th }}$ column the C-plane view at a depth of 50 mm. (a) Images acquired in the static state, shown as benchmark. (b) Images acquired in the moving state without MoCo, with motion in the axial direction at a velocity of $10 \mathrm{~cm} / \mathrm{s}$. (c) Images acquired in the moving state with MoCo.

DWC are similar to those of $5 \times 5$ DWC, i.e., blurring and deterioration of image quality without $\mathrm{MoCo}$, and restoration with $\mathrm{MoCo}$, as illustrated on the $1^{\text {st }}$ and $2^{\text {nd }}$ rows of Fig. 8, respectively. For the $3 \mathrm{MPT}$ beamforming, the image in the azimuth dimension (where the beams are diverging) without MoCo showed some blurring and a global offset in the axial direction, as illustrated in Fig. 8(c1). In the elevation dimension, where the beams behave as MLT, stitching artifacts occur, showing increased displacements from the edge to the center in each sub-sector, due to the "edge-to-center" scan sequence, as illustrated in Fig. 8(c2). However, the images are significantly restored after applying the proposed MoCo strategy, as illustrated on the final row of Fig. 8.

The MoCo results in the presence of noise are shown in Fig. 9(a) and 9(b), with the noise added leading to an SNR of $10 \mathrm{~dB}$ before MoCo. Fig. 9(a) gives the results of $9 \times 9$ DWC with the Diagonal sequence, with Fig. 9(a1) to 9(a4) presenting the images without MoCo, the estimated velocities, the displacements between the last and the first volumes in the transmission process, and the images with MoCo, respectively. The estimated velocities and displacements are homogenous and smooth in the phantom in both dimensions. The results of 3MPT-2MLA-5CMP are given in Fig. 9(b). The velocities of $3 \mathrm{MPT}$ show more variations compared with those of $9 \times 9$ DWC, mainly due to a much shorter ensemble length in the slow-time direction for the Doppler velocity estimation (i.e., 5 versus 81 points, for the MPT and DWC sequences respectively). The displacements are also obtained between the last and the first transmit events. The displacements in the azimuth dimension are equally homogeneous and smooth. However, in the elevation-axial plane, the displacements increase from the edge to the center within the sub-sectors, which matches the "edge-to-center" scan sequence in this dimension. 


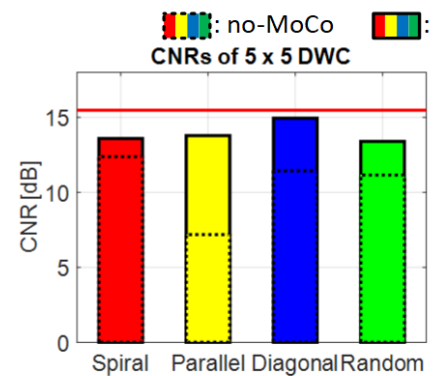

(a1)

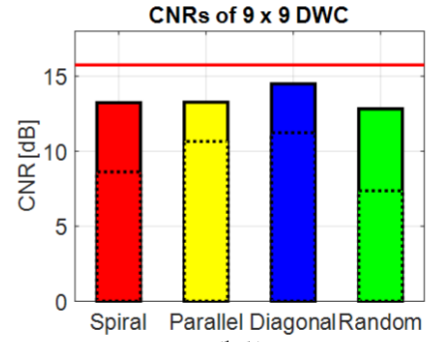

(b1)

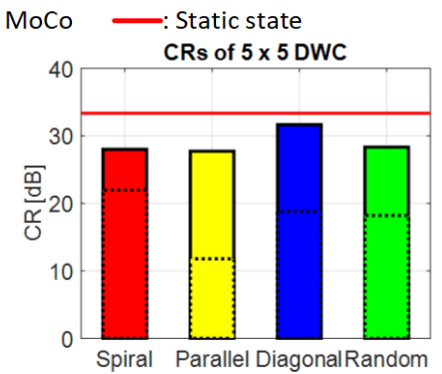

(a2)

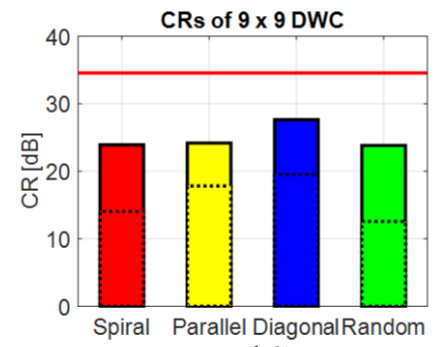

(b2)

Fig. 7. Quantitative CNRs and CRs of the $5 \times 5$ and $9 \times 9$ DWC, both with the four sequences (i.e., the Spiral, Parallel, Diagonal and Random sequences) being tested. (a1) CNRs of the four $5 \times 5$ DWC sequences. (a2) CRs of the four $5 \times 5$ DWC sequences. (b1) CNRs of the four $9 \times 9$ DWC sequences. (b2) CRs of the four $9 \times 9 \mathrm{DWC}$ sequences. The red lines indicate the results in the static state, shown as benchmark. The bars in dashed/solid lines indicate the results without/with MoCo.

The quantitative CNRs and CRs of both sequences at different SNR levels are presented in Fig. 9(c1) and 9(c2), respectively. The condition of infinite SNR means that there was no noise added to the simulations. It can be seen that in the static state, $9 \times 9$ DWC obtains slightly higher CNR and CR when compared with 3MPT-2MLA-5CMP (CNR: $15.75 \mathrm{~dB}$ versus $15.59 \mathrm{~dB}$; CR: $34.55 \mathrm{~dB}$ versus $33.16 \mathrm{~dB}$ ). However, when motion is present and MoCo is applied, 3MPT-2MLA-5CMP with MoCo outperforms $9 \times 9$ DWC with the Diagonal sequence with MoCo, showing higher levels of CNRs and CRs in all SNR conditions. Meanwhile, the MoCo strategies become invalid in very low SNR condition (i.e., -20 dB), as illustrated in Fig. 9(c2).

\section{DISCUSSION}

In this study, different beamforming approaches towards high frame rate volumetric ultrasound imaging were contrasted in particular with respect to their sensitivity to motion-induced artifacts. The major conclusions of this study are: i) MoCo is effective for both 3D DWC and MPT; ii) MoCo for 3D DWC is most effective when using a Diagonal transmit sequence; and iii) the 3MPT-2MLA-5CMP with MoCo outperforms $9 \times 9$ DWC with the Diagonal sequence with MoCo in image quality for a competitive temporal resolution.

The "round-trip" scan scheme is important in the proposed Doppler-based velocity estimation. In both 3D DWC and MPT, the PSFs are rotating during the transmission process. For DWC, the PSFs are rotating in $3 \mathrm{D}$ according to the positions of the virtual sources, while for MPT, the PSFs are rotating in 2D planes as compounding is performed only in the azimuth dimension. Such rotation makes the phase of the PSF's side

lobes incoherent, which is the reason why compounding is effective. However, such rotation also induces unwanted velocities and these rotation-induced velocities are involved in the estimated Doppler velocities, thus resulting in a biased estimation. When using the "round-trip" scan sequence, the directions of the rotation-induced velocities are opposite in the "forward" and "backward" trips. As a result, the product of the auto-correlation coefficients in the "forward" and "backward" trips can be used to remove these rotation-induced velocities effectively, leaving the true velocities only [44]. It should be noted that the "round-trip" scan sequence covers the imaged volume twice, so two volumetric images could be reconstructed after $\mathrm{MoCo}$, without sacrificing the volume rate.

The Diagonal sequence performs the best for 3D DWC as it provides a more balanced time-lag in the azimuth-axial plane, the elevation-axial plane, and the two diagonal planes in sequential firings, thus leading to more isotropic image qualities in those cross-sections [see Fig. 4(c) and 7]. This is also indirectly proven in Fig. 6 (i.e., the images were acquired from $5 \times 5$ DWC with Parallel sequence), where the image in the elevation-axial plane shows higher level of side lobes in the cystic region and outside the cubic phantom than that in the azimuth-axial plane, due to a five times larger time lag of sequential firings in the elevation dimension, as illustrated in Fig. 6(b1) and 6(b2) respectively. However, it should be noted that the Parallel sequence performed slightly better than the Spiral and Random sequences in terms of CNR measurement, as illustrated in Fig. 7. In fact, the patterns of the Diagonal and Parallel sequences show some similarities, except that the former is transmitted along the diagonal direction, while the latter in the azimuth direction. This conclusion is in agreement with the recent findings in [46], where a dedicated sequence similar to our Parallel sequence was used for MoCo of a series of steered 3D diverging waves (see Fig. 1 in [46]).

The 3MPT-2MLA-5CMP sequence shows slightly lower $\mathrm{CNR}$ and $\mathrm{CR}$ in the static state compared with $9 \times 9 \mathrm{DWC}$, mainly due to the potential crosstalk artifacts caused by the interaction in between the MPT beams, as illustrated in Fig. 2(c4) and 9(c), as well as less compounding made in the azimuth dimension. However, when motion exists and MoCo is applied, the 3MPT sequence with MoCo shows better performance [see Figs. 8 and 9]. Moreover, $5 \times 5$ DWC with MoCo generally obtains higher CNRs and CRs than $9 \times 9$ DWC when motion exists [see Fig. 7]. The inferior performance of 9 $\times 9 \mathrm{DWC}$ with MoCo is probably due to underestimation of the Doppler velocity. From a signal processing perspective, the lag-one auto-correlation algorithm estimates the mean slow-time frequency. Meanwhile, a finite length of the axial gate $(\sim 0.4 \mathrm{~mm}$ in this study) is commonly used in Doppler algorithms, which also averages the fast-time velocity estimates. Furthermore, the short transmit pulse (1.5 cycles) used in this study might also be a factor to cause variance in the velocity estimation, as conventionally $6 \sim 8$ cycles are used in ultrasound Doppler imaging. The underestimation of the Doppler velocity can also be found in the literature on blood flow imaging [52]-[55]. In particular, this underestimation is significantly amplified during the massive compounding 


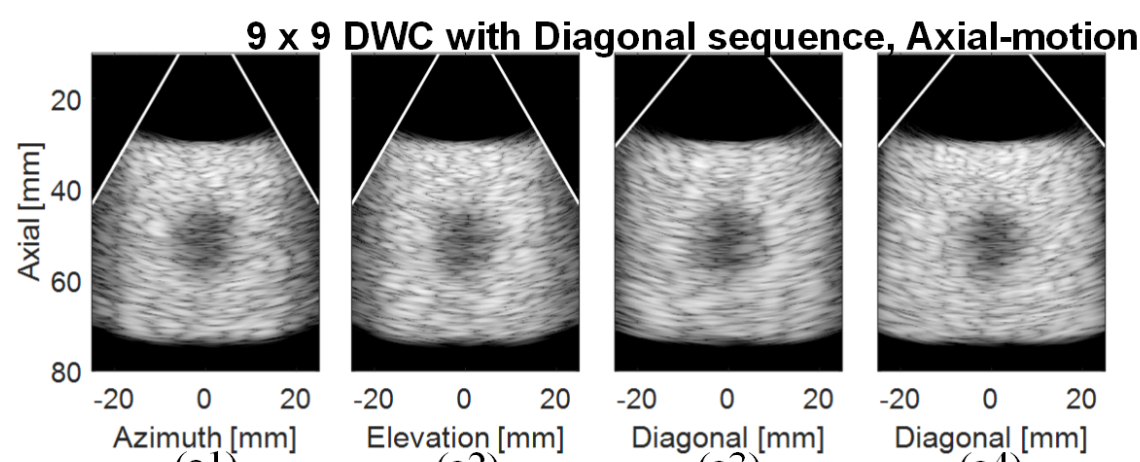

(a1) (a2) (a3) (a4)

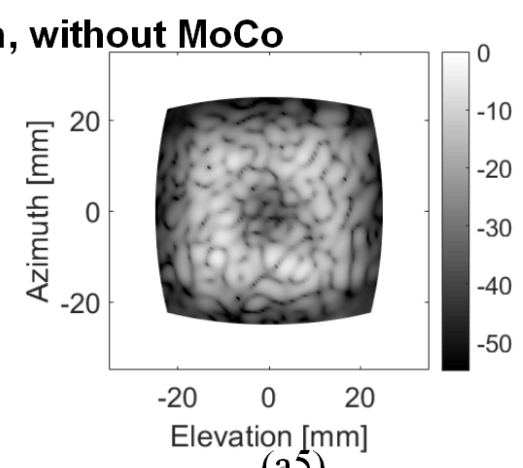

(a5)

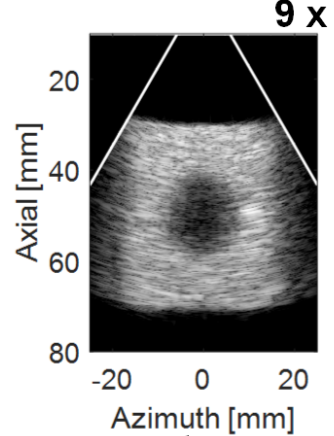

(b1)

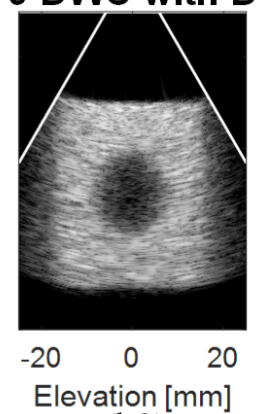

(b2)

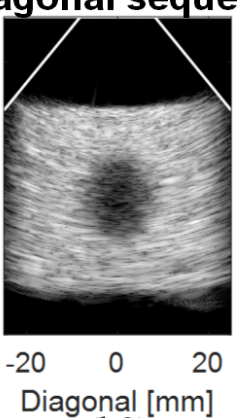

(b3)

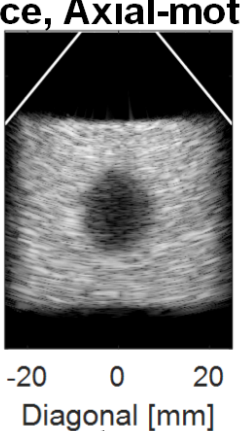

(b4)

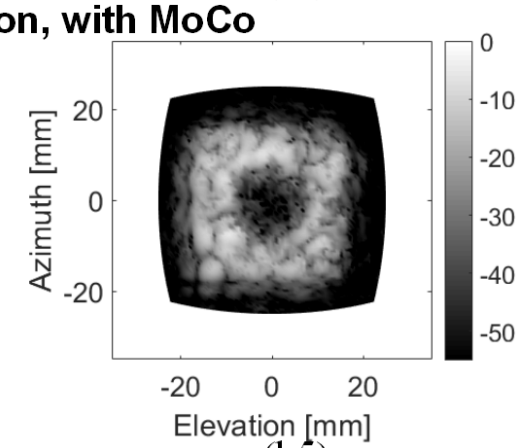

(b5)

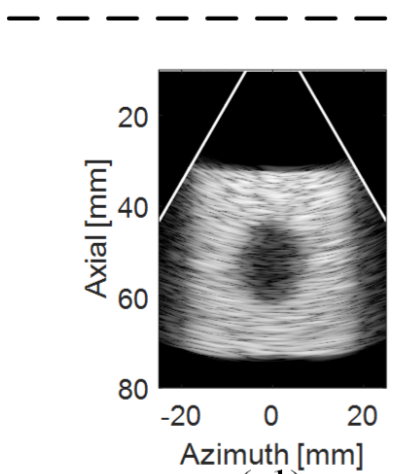

(c1)

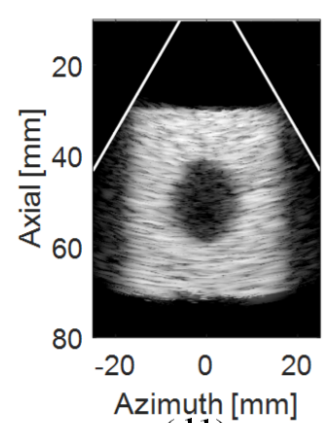

(d)

3MPT-2MLA- $\overline{\mathrm{MCMP}}$, Axial-motion, without MoCo

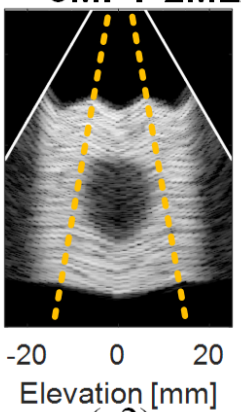

(c2)

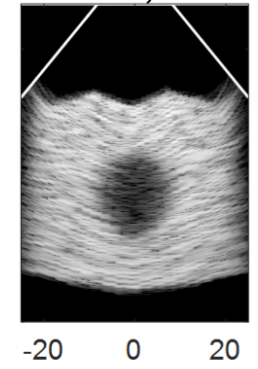

Diagonal [mm]

(c3)

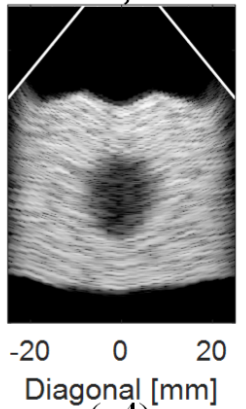

(c4)

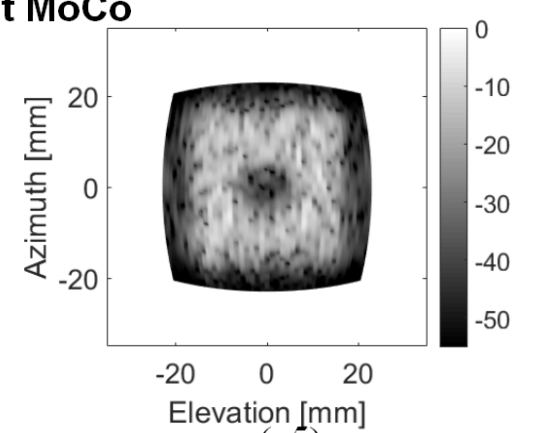

(c5)

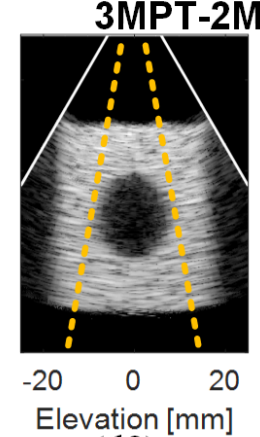

(d2)

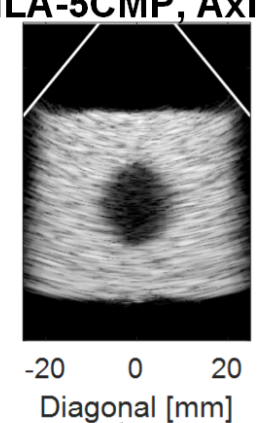

(d3)

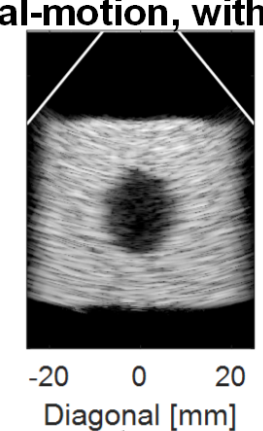

(d4)

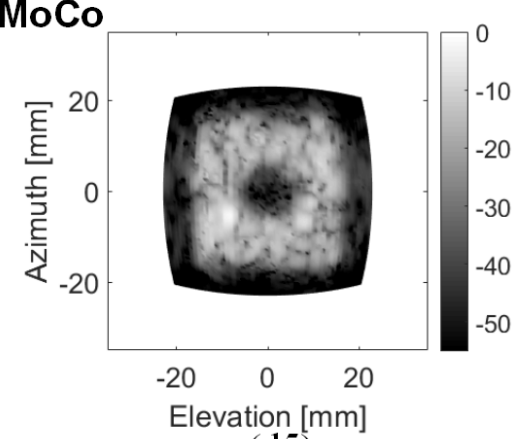

(d5)

Fig. 8. Images acquired by $9 \times 9$ DWC with the Diagonal sequence and the 3MPT-2MLA-5CMP in the static and moving state, respectively. The $1^{\text {st }}$ column shows the azimuth-axial plane, the $2^{\text {nd }}$ column the elevation-axial plane, the $3^{\text {rd }}$ and $4^{\text {th }}$ columns the two planes in the diagonal dimensions, and the $5^{\text {th }}$ column the C-plane view at a depth of $50 \mathrm{~mm}$. (a) Images acquired from $9 \times 9 \mathrm{DWC}$ in the moving state without MoCo, with motion in the axial direction at a velocity of 10 $\mathrm{cm} / \mathrm{s}$. (b) Images acquired from $9 \times 9$ DWC in the moving state with MoCo. (c) Images acquired from 3MPT-2MLA-5CMP in the moving state without MoCo, with motion in the axial direction at a velocity of $10 \mathrm{~cm} / \mathrm{s}$. (d) Images acquired from 3MPT-2MLA-5CMP in the moving state with MoCo. The yellow dashed lines indicate the edges of the scanning sub-sectors in the elevation dimension of the $3 \mathrm{MPT}$ sequence.

operation, e.g., with $9 \times 9$ virtual sources. Nevertheless, such bias is less critical in the 3MPT-2MLA-5CMP because only 5 planar diverging waves are compounded within each of the MPT planes. As for $5 \times 5 \mathrm{DWC}$, the number of compounds are 


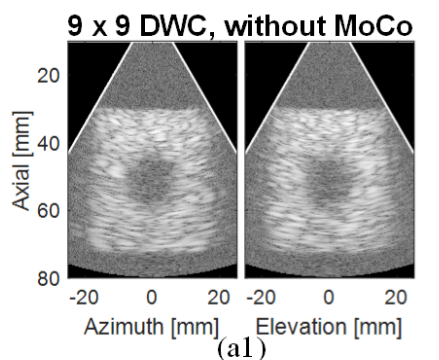

(a1)

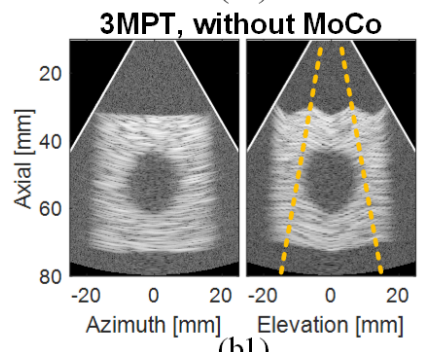

(b1)

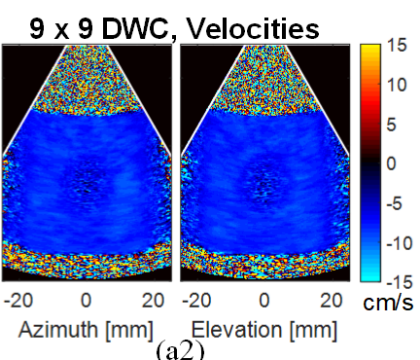

(a2)

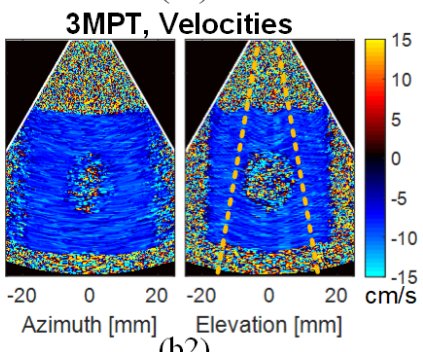

(b2)

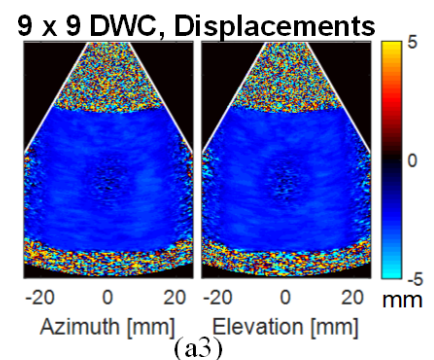

(a3)

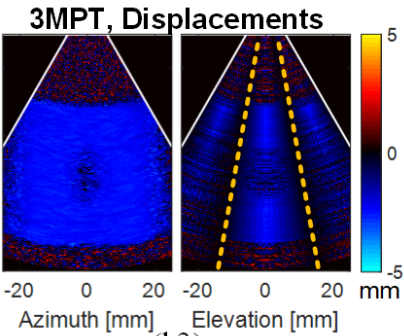

(b3)

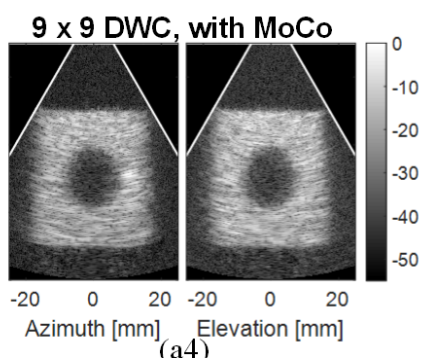

(a4)

3MPT, with MoCo

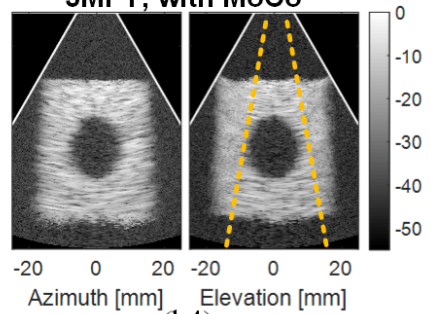

(b4)

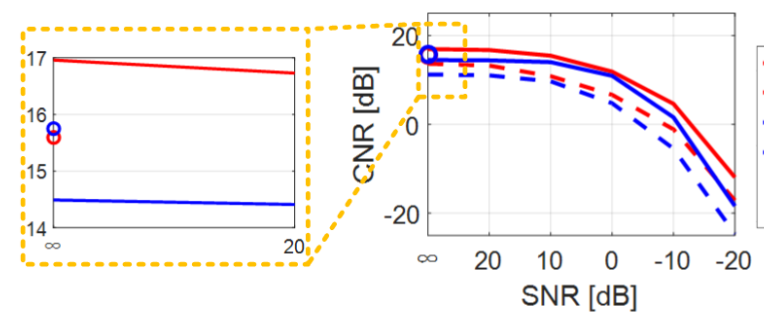

(c1)

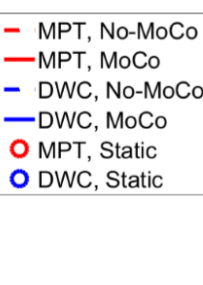

- MPT, MoCo

-DWC, MoCo

MPT, Static

DWC, Static

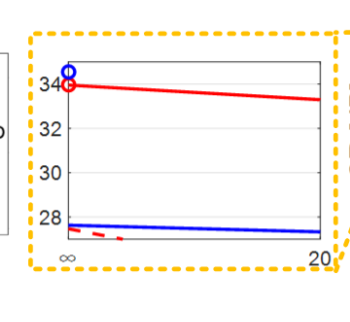

(c2)

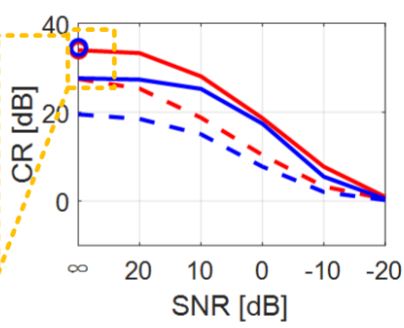

Fig. 9. Images and quantitative CNRs and CRs in $9 \times 9$ DWC with the Diagonal sequence and the proposed 3MPT-2MLA-5CMP sequence in the presence of noise. (a) Images acquired from $9 \times 9 \mathrm{DWC}$ at an SNR of $10 \mathrm{~dB}$, with (a1) the images without MoCo, (a2) the estimated Doppler velocities, (a3) the displacements between the last and the first volumes in the transmission process, and (a4) the images with MoCo. (b) Images acquired from 3MPT-2MLA-5CMP at an SNR of $10 \mathrm{~dB}$, with (b1) the images without MoCo, (b2) the estimated Doppler velocities, (b3) the displacements between the last and the first transmission events, and (b4) the images with MoCo. The yellow dashed lines indicate the edges of the scanning sub-sectors in the elevation dimension of the $3 \mathrm{MPT}$ sequence. (c) The quantitative CNRs and CRs of the two sequences in the presence of different levels of noise. The condition of infinite SNR means that there is no noise added to the simulations.

thus markedly smaller when compared with $9 \times 9$ DWC. As a result, such bias has a smaller impact on $5 \times 5 \mathrm{DWC}$ and $3 \mathrm{MPT}$.

MPT is intrinsically a plane-by-plane beamforming approach, so scanning is needed in the dimension where the beams are focused. As a result, its potential capability of further increasing the volume rate is limited when compared with 3D DWC. The volume rate of MPT could be increased by increasing the simultaneously transmitted beams, or by reducing the number of compounds made in the azimuth dimension. However, the former could introduce more crosstalk artifacts, while the latter might lead to an unbalanced image quality in the azimuth and elevation dimensions. It should be noted that although $5 \times 5 \mathrm{DWC}$ has a higher volume rate than 3MPT-2MLA-5CMP in this study, the latter on the contrary, provides better image quality. When compared with 9 $\times 9$ DWC, 3MPT-2MLA-5CMP not only has competitive volume rate, but also provides better image quality when motion exists and MoCo is applied.

In this study, the beams of 3D DWC, and MPT in the azimuth dimension are both transmitted from a sub-aperture without steering, and compounding is performed by moving the sub-aperture without changing the beam profiles similar to what was done in [29]. Such setup is different from what was used in [30], [44] and [46], where the 2D/3D diverging waves are transmitted using the full aperture, and compounding is performed by steering the beams. Sub-aperture transmission without beam steering is used in this study in order not to introduce extra side lobes and/or grating lobes caused by beam steering in the limited-sized matrix array. Particularly in MPT, the planar diverging waves already have steering in the elevation dimension [see Fig. 3(b)]. As a result, adding extra steering in the azimuth dimension would increase the risk of introducing more side lobes and/or grating lobes. Furthermore, it is recently demonstrated that translating the active sub-aperture over the phased array rather than tilting the transmit beams is beneficial for the 2D DWC imaging [51].

One of the limitations in this study is that the size of the simulated matrix array $\left(\sim 1 \mathrm{~cm}^{2}\right)$ is much smaller than that of the conventional phased array ( $\sim 2 \mathrm{~cm}$ in length), leading to worse lateral resolution in the azimuth and elevation dimensions. Thus imaging based on such matrix array is less sensitive to lateral motion (i.e., motion in the azimuth or elevation directions). As a result, only MoCo along the beamformed lines is performed in the present study.

Another limitation is that the simulated motion is rigid at a constant velocity. However, the anatomical structure and motion of the heart are very complex, and the echo signals will be significantly affected by surrounding tissues. As a result, the 
simulated moving phantom in this study is only a simplified model for the heart motion. However, the objective of this study is to propose the 3D MoCo strategies and perform their preliminary verification. Experimental validation of the methods on tissue-mimicking phantoms are the topic of future work.

Finally, it should be noted that we do not simulate 3D MLT with Doppler-based MoCo strategy in the present study, considering that several transmissions (typically 8 12) should be repeated in one direction for a conventional Doppler estimation. Such imaging setup requires more than 1,000 transmission events (i.e. 144 firings are required for the 16MLT-4MLA setup to cover the imaging volume of 90-by-90 lines) [47], thus seriously compromising the temporal resolution to a level lower than $5 \mathrm{~Hz}$, thereby making the comparison with DWC and MPT unfair.

\section{CONCLUSION}

In this paper, Doppler-based MoCo strategies are proposed and demonstrated to be effective in MPT and 3D DWC via computer simulations. For 3MPT-2MLA-5CMP, a 2-step MoCo strategy is implemented in order to significantly reduce motion artifacts in both azimuth and elevation dimensions. For the 3D DWC, a "round-trip" scan sequence followed by a Doppler-based MoCo strategy is proposed to directly compensate motion in 3D. Both the qualitative and quantitative results demonstrate that: i) the Diagonal sequence is a better choice for 3D DWC with MoCo; ii) 3MPT-2MLA-5CMP with MoCo outperforms $9 \times 9$ DWC with Diagonal sequence with $\mathrm{MoCo}$, thus allows to reconstruct high-quality volumetric images that are acquired at real time and are minimally affected by motions. Experimental validations of these results are the topic of future work.

\section{REFERENCES}

[1] J. Provost, W. N. Lee, K. Fujikura, and E. E. Konofagou, "Electromechanical Wave Imaging of Normal and Ischemic Hearts in Vivo," IEEE Trans. Medical Imaging, vol. 29, pp. 625-635, 2010.

[2] E. Konofagou, W. N. Lee, J. Luo, J. Provost, and J. Vappou, "Physiologic cardiovascular strain and intrinsic wave imaging," Annual Review of Biomedical Engineering, vol. 13, pp. 477-505, 2011.

[3] W.-N. Lee, M. Pernot, M. Couade, E. Messas, P. Bruneval, A. Bel, et al., "Mapping myocardial fiber orientation using echocardiography-based shear wave imaging," IEEE Trans. Medical Imaging, vol. 31, pp. 554-562, 2012.

[4] M. Correia, J. Provost, S. Chatelin, O. Villemain, M. Tanter, and M. Pernot, "Ultrafast harmonic coherent compound (UHCC) imaging for high frame rate echocardiography and shear-wave elastography," IEEE Trans. Ultrasonics, Ferroelectrics, and Frequency Control, vol. 63, pp. 420-431, 2016.

[5] A. J. Engel, H. H. Hsu, P. Song, and G. R. Bashford, "Cardiac atrial kick shear wave elastography with ultrafast diverging wave imaging: An in vivo pilot study," in Proc. IEEE International Ultrasonics Symposium (IEEE IUS), 2017, pp. 1-4.

[6] R. C. Houck, J. Cooke, and E. A. Gill, "Three-dimensional echo: transition from theory to real-time, a technology now ready for prime time," Current Problems in Diagnostic Radiology, vol. 34, pp. 85-105, 2005.

[7] R. M. Lang, V. Mor-Avi, L. Sugeng, P. S. Nieman, and D. J. Sahn, "Three-dimensional echocardiography: the benefits of the additional dimension," Journal of the American College of Cardiology, vol. 48, pp.
2053-2069, 2006.

[8] L. P. Badano, R. M. Lang, and J. L. Zamorano, Textbook of Real-time Three Dimensional Echocardiography: Springer, 2011.

[9] M. Cikes, L. Tong, G. R. Sutherland, and J. D'hooge, "Ultrafast cardiac ultrasound imaging: technical principles, applications, and clinical benefits," JACC: Cardiovascular Imaging, vol. 7, pp. 812-823, 2014.

[10] R. Mallart and M. Fink, "Improved imaging rate through simultaneous transmission of several ultrasound beams," in Proc. SPIE, 1992, pp. 120-130.

[11] L. Tong, H. Gao, and J. D'hooge, "Multi-transmit beam forming for fast cardiac imaging-a simulation study," IEEE Trans. Ultrasonics, Ferroelectrics, and Frequency Control, vol. 60, pp. 1719-1731, 2013.

[12] L. Tong, A. Ramalli, R. Jasaityte, P. Tortoli, and J. D'hooge, "Multi-transmit beam forming for fast cardiac imaging - Experimental validation and in vivo application," IEEE Trans. Medical Imaging, vol. 33, pp. 1205-1219, 2014.

[13] L. Tong, A. Ortega, H. Gao, and J. D'hooge, "Fast three-dimensional ultrasound cardiac imaging using multi-transmit beam forming: A simulation study," in Proc. IEEE International Ultrasonics Symposium (IEEE IUS), 2013, pp. 1456-1459.

[14] B. Denarie, T. Bjastad, and H. Torp, "Multi-line transmission in 3-D with reduced crosstalk artifacts: A proof of concept study," IEEE Trans. Ultrasonics, Ferroelectrics, and Frequency Control, vol. 60, pp. 1708-1718, 2013.

[15] P. Santos, L. Tong, A. Ortega, L. Løvstakken, E. Samset, and J. D'hooge, "Safety of Multi-Line Transmit beam forming for fast cardiac imaging-a simulation study," in Proc. IEEE International Ultrasonics Symposium (IEEE IUS), 2014, pp. 1199-1202.

[16] P. Santos, L. Tong, A. Ortega, L. Løvstakken, E. Samset, and J. D’hooge, "Acoustic output of multi-line transmit beamforming for fast cardiac imaging: A simulation study," IEEE Trans. Ultrasonics, Ferroelectrics, and Frequency Control, vol. 62, pp. 1320-1330, 2015.

[17] G. Matrone, A. Ramalli, A. S. Savoia, P. Tortoli, and G. Magenes, "High frame-rate, high resolution ultrasound imaging with multi-line transmission and filtered-delay multiply and sum beamforming," IEEE Trans. Medical Imaging, vol. 36, pp. 478-486, 2017.

[18] A. Rabinovich, A. Feuer, and Z. Friedman, "Multi-line transmission combined with minimum variance beamforming in medical ultrasound imaging," IEEE Trans. Ultrasonics, Ferroelectrics, and Frequency Control, vol. 62, pp. 814-827, 2015.

[19] F. Prieur, B. Dénarié, A. Austeng, and H. Torp, "Correspondence-Multi-line transmission in medical imaging using the second-harmonic signal," IEEE Trans. Ultrasonics, Ferroelectrics, and Frequency Control, vol. 60, pp. 2682-2692, 2013.

[20] Zurakhov G, Tong L, Ramalli A, et al. "Multi Line Transmit Beamforming Combined with Adaptive Apodization," IEEE Trans. Ultrasonics, Ferroelectrics, and Frequency Control, 2018.

[21] A. Ortega, L. Tong, P. Santos, B. Heyde, and J. D'hooge, "Fast volumetric cardiac ultrasound: a comparison of different multi-line transmit setups by computer simulation," in Proc. IEEE International Ultrasonics Symposium (IEEE IUS), 2014, pp. 1195-1198.

[22] A. Ortega, J. Provost, L. Tong, P. Santos, B. Heyde, M. Pernot, et al., "A Comparison of the Performance of Different Multiline Transmit Setups for Fast Volumetric Cardiac Ultrasound," IEEE Trans. Ultrasonics, Ferroelectrics, and Frequency Control, vol. 63, pp. 2082-2091, 2016.

[23] K. L. Gammelmark and J. A. Jensen, "2-D tissue motion compensation of synthetic transmit aperture images," IEEE Trans. Ultrasonics, Ferroelectrics, and Frequency Control, vol. 61, pp. 594-610, 2014.

[24] L. Tong, A. Ramalli, P. Tortoli, G. Fradella, S. Caciolli, J. Luo, et al., "Wide-angle tissue Doppler imaging at high frame rate using multi-line transmit beamforming: An experimental validation in vivo," IEEE Trans. Medical Imaging, vol. 35, pp. 521-528, 2016.

[25] D. P. Shattuck, M. D. Weinshenker, S. W. Smith, and O. T. von Ramm, "Explososcan: A parallel processing technique for high speed ultrasound imaging with linear phased arrays," The Journal of the Acoustical Society of America, vol. 75, pp. 1273-1282, 1984.

[26] M. Tanter and M. Fink, "Ultrafast imaging in biomedical ultrasound," IEEE Trans. Ultrasonics, Ferroelectrics, and Frequency Control, vol. 61, pp. 102-119, 2014.

[27] G. Montaldo, M. Tanter, J. Bercoff, N. Benech, and M. Fink, "Coherent plane-wave compounding for very high frame rate ultrasonography and 
transient elastography," IEEE Trans. Ultrasonics, Ferroelectrics, and Frequency Control, vol. 56, pp. 489-506, 2009.

[28] M. Couade, M. Pernot, M. Tanter, E. Messas, A. Bel, M. Ba, et al., "Ultrafast imaging of the heart using circular wave synthetic imaging with phased arrays," in Proc. IEEE International Ultrasonics Symposium (IEEE IUS), 2009, pp. 515-518

[29] C. Papadacci, M. Pernot, M. Couade, M. Fink, and M. Tanter, "High-contrast ultrafast imaging of the heart," IEEE Trans. Ultrasonics, Ferroelectrics, and Frequency Control, vol. 61, pp. 288-301, 2014.

[30] H. Hasegawa and H. Kanai, "High-frame-rate echocardiography using diverging transmit beams and parallel receive beamforming," Journal of Medical Ultrasonics, vol. 38, pp. 129-140, 2011.

[31] J. Provost, C. Papadacci, J. E. Arango, M. Imbault, M. Fink, J.-L. Gennisson, et al., "3D ultrafast ultrasound imaging in vivo," Physics in Medicine and Biology, vol. 59, p. L1, 2014.

[32] J.-1. Gennisson, J. Provost, T. Deffieux, C. Papadacci, M. Imbault, M. Pernot, et al., "4-D ultrafast shear-wave imaging," IEEE Trans. Ultrasonics, Ferroelectrics, and Frequency Control, vol. 62, pp. 1059-1065, 2015

[33] J. Wang and J.-y. Lu, "Motion artifacts of extended high frame rate imaging," IEEE Trans. Ultrasonics, Ferroelectrics, and Frequency Control, vol. 54, pp. 1303-1315, 2007.

[34] B. Denarie, T. A. Tangen, I. K. Ekroll, N. Rolim, H. Torp, T. Bjåstad, et al., "Coherent plane wave compounding for very high frame rate ultrasonography of rapidly moving targets," IEEE Trans. Medical Imaging, vol. 32, pp. 1265-1276, 2013.

[35] L. F. Nock and G. E. Trahey, "Synthetic receive aperture imaging with phase correction for motion and for tissue inhomogeneities. I. Basic principles," IEEE Trans. Ultrasonics, Ferroelectrics, and Frequency Control, vol. 39, pp. 489-495, 1992.

[36] G. E. Trahey and L. F. Nock, "Synthetic receive aperture imaging with phase correction for motion and for tissue inhomogeneities. II. Effects of and correction for motion," IEEE Trans. Ultrasonics, Ferroelectrics, and Frequency Control, vol. 39, pp. 496-501, 1992.

[37] M. Karaman, H. S. Bilge, and M. O'Donnell, "Adaptive multi-element synthetic aperture imaging with motion and phase aberration correction," IEEE Trans. Ultrasonics, Ferroelectrics, and Frequency Control, vol. 45, pp. 1077-1087, 1998.

[38] K. Kim, J. Hwang, J. Jeong, and T. Song, "An efficient motion estimation and compensation method for ultrasound synthetic aperture imaging," Ultrasonic Imaging, vol. 24, pp. 81-99, 2002.

[39] M. Tanter, J. Bercoff, L. Sandrin, and M. Fink, "Ultrafast compound imaging for 2-D motion vector estimation: Application to transient elastography," IEEE Trans. Ultrasonics, Ferroelectrics, and Frequency Control, vol. 49, pp. 1363-1374, 2002.

[40] K. L. Gammelmark and J. A. Jensen, "Duplex synthetic aperture imaging with tissue motion compensation," in Proc. IEEE International Ultrasonics Symposium (IEEE IUS), 2003, pp. 1569-1573.

[41] M.-H. Bae, B.-S. Kim, M.-K. Jeong, J.-H. Ham, D.-Y. Kim, W.-Y. Lee, et al., "P2B-3 A New Motion Estimation and Compensation Method for Real-Time Ultrasonic Synthetic Aperture Imaging," in Proc. IEEE International Ultrasonics Symposium (IEEE IUS), 2007, pp. 1511-1513.

[42] B. Y. Yiu, I. K. Tsang, and C. Alfred, "A modified synthetic aperture imaging approach with axial motion compensation," in Proc. IEEE International Ultrasonics Symposium (IEEE IUS), 2008, pp. 1254-1257.

[43] K.-Y. Ho, Y.-H. Chen, C.-Z. Zhan, and A.-Y. A. Wu, "VLSI implementation of real-time motion compensated beamforming in synthetic transmit aperture imaging," in Proc. IEEE International Symposium on Circuits and Systems (ISCAS), 2013, pp. 1893-1896.

[44] J. Porée, D. Posada, A. Hodzic, F. Tournoux, G. Cloutier, and D. Garcia, "High-frame-rate echocardiography using coherent compounding with Doppler-based motion-compensation," IEEE Trans. Medical Imaging, vol. 35, pp. 1647-1657, 2016.

[45] Joos P, Porée J, Liebgott H, et al. "High-frame-rate speckle tracking echocardiography," IEEE Trans. Ultrasonics, Ferroelectrics, and Frequency Control, 2018.

[46] P. Joos, H. Liebgott, F. Varray, L. Petrusca, D. Garcia, D. Vray and B. Nicolas. "High-Frame-Rate 3-D Echocardiography Based on Motion Compensation: An in Vitro Evaluation," in Proc. IEEE International Ultrasonics Symposium (IEEE IUS), 2017, pp. 1-4.

[47] Y. Chen, L. Tong, A. Ortega, J. Luo, and J. D'hooge, "Feasibility of
Multiplane-Transmit Beamforming for Real-Time Volumetric Cardiac Imaging: A Simulation Study," IEEE Trans. Ultrasonics, Ferroelectrics, and Frequency Control, vol. 64, pp. 648-659, 2017.

[48] J. A. Jensen, "Field: A program for simulating ultrasound systems," in 10th Nordic-Baltic Conference on Biomedical Imaging Published in Medical \& Biological Engineering \& Computing, Vol. 4, Supplement 1, Part 1: 351--353, 1996.

[49] J. A. Jensen and N. B. Svendsen, "Calculation of pressure fields from arbitrarily shaped, apodized, and excited ultrasound transducers," IEEE Trans. Ultrasonics, Ferroelectrics, and Frequency Control, vol. 39, pp. 262-267, 1992

[50] S. F. Nagueh, K. J. Middleton, H. A. Kopelen, W. A. Zoghbi, and M. A. Quiñones, "Doppler tissue imaging: a noninvasive technique for evaluation of left ventricular relaxation and estimation of filling pressures," Journal of the American College of Cardiology, vol. 30, pp. 1527-1533, 1997.

[51] V. Komini, P. Santos, and J. D'hooge. "Diverging Wave Compounding: Direct Comparison of Two Popular Approaches," in Proc. IEEE International Ultrasonics Symposium (IEEE IUS), 2017, pp. 1-4.

[52] J. Udesen, F. Gran, K. L. Hansen, J. A. Jensen, C. Thomsen, and M. B. Nielsen, "High frame-rate blood vector velocity imaging using plane waves: Simulations and preliminary experiments," IEEE Trans. Ultrasonics, Ferroelectrics, and Frequency Control, vol. 55, pp. 1729-1743, 2008.

[53] I. K. Ekroll, A. Swillens, P. Segers, T. Dahl, H. Torp, and L. Lovstakken, "Simultaneous quantification of flow and tissue velocities based on multi-angle plane wave imaging," IEEE Trans. Ultrasonics, Ferroelectrics, and Frequency Control, vol. 60, pp. 727-738, 2013.

[54] S. Fadnes, I. K. Ekroll, S. A. Nyrnes, H. Torp, and L. Lovstakken, "Robust angle-independent blood velocity estimation based on dual-angle plane wave imaging," IEEE Trans. Ultrasonics, Ferroelectrics, and Frequency Control, vol. 62, pp. 1757-1767, 2015.

[55] B. Y. Yiu and C. Alfred, "Least-squares multi-angle Doppler estimators for plane-wave vector flow imaging," IEEE Trans. Ultrasonics, Ferroelectrics, and Frequency Control, vol. 63, pp. 1733-1744, 2016.

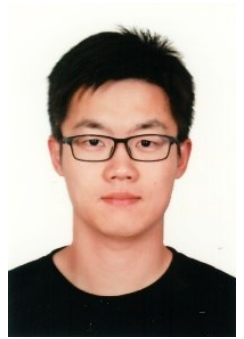

Yinran Chen (S'16) was born in Fujian, China, in 1991. He received the bachelor's degree in biomedical engineering from Tsinghua University, Beijing, China, in 2014, where he is currently pursuing the Ph.D. degree with the Department of Biomedical Engineering. He joined the Cardiovascular Imaging and Dynamics Laboratory, University of Leuven, Leuven, Belgium, as a Visiting Scholar, in 2015. His current research interests include ultrasound beamforming and signal processing for high-frame-rate imaging.

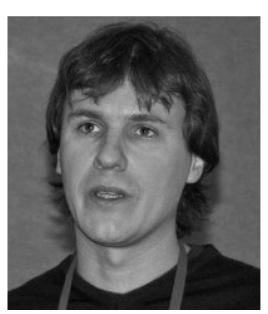

Jan D'hooge (M'98) was born in Sint-Niklaas, Belgium, in 1972. He received the M.Sc. and Ph.D. degrees in physics from the University of Leuven, Leuven, Belgium, in 1994 and 1999, respectively. His dissertation was focused on the interaction of ultrasonic waves and biological tissues by means of computer simulation. He was a Post-Doctoral Researcher with the Medical Imaging Computing Laboratory, University of Leuven, where he became acquainted with general problems in medical imaging, such as elastic registration, segmentation, shape analysis, and data acquisition problems related to other modalities, particularly in MRI. In 2006, he joined the Department of Cardiovascular Diseases with the Medical Faculty, Katholieke Universiteit Leuven, Leuven, as an 
Associate Professor. Since 2009, he has been a part-time Visiting Professor with the Norwegian Institute of Science and Technology, Trondheim, Norway. He has coauthored over 150 peer-reviewed papers, contributed to eight books, and coedited one book. His current research interests include myocardial tissue characterization, deformation imaging, and cardiac patho-physiology.

Dr. D'hooge is a member of the Acoustical Society of America and the European Association of Echocardiography. In 1999, he was a recipient of the Young Investigator Award of the Belgian Society of Echocardiography. In 2000, he was nominated for the Young Investigator Award of the European Society of Echocardiography. He was the Chair of the Ultrasound Conference of the SPIE Medical Imaging Symposium from 2008 to 2011, the Technical Vice-Chair of the IEEE Ultrasonics Symposium from 2008 to 2012, and the Technical Chair of the IEEE Ultrasonics Symposium in 2014. He was an elected AdCom Member of the IEEE-UFFC Society from 2010 to 2012 .

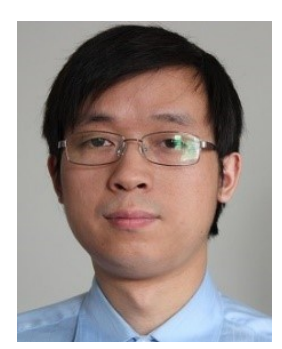

Jianwen Luo (S'02-M'06-SM'14) received the B.S. and Ph.D. (with honors) degrees in Biomedical Engineering from Tsinghua University, Beijing, China, in 2000 and 2005, respectively. He was a Postdoctoral Research Scientist from 2005 to 2009, and an Associate Research Scientist from 2009 to 2011, in the Department of Biomedical Engineering, Columbia University, New York, NY. He became a Professor in the Department of Biomedical Engineering and the Center for Biomedical Imaging Research at Tsinghua University in 2011. He was enrolled in the Thousand Young Talents Program of China in 2012, and received the Excellent Young Scientists Fund from the National Natural Science Foundation of China (NSFC) in 2013. He was supported by the Young Scientists Project of National Key R\&D Program of China in 2016. His research interests include ultrasound imaging and fluorescence imaging. He has authored or coauthored over 120 peer-reviewed papers in international journals, 50 conference proceedings papers, and 130 conference abstracts. He serves as an advisory editorial board member of Journal of Ultrasound in Medicine, and a member of the IEEE Engineering in Medicine and Biology Society (EMBS) Technical Committee on Biomedical Imaging and Image Processing (BIIP). 